\title{
10 Spreadsheets: just smart calculators or a new paradigm for thinking about mathematical structures?
}

\author{
Erich Neuwirth \\ Computer Supported Didactics Working Group \\ University of Vienna
}

\begin{abstract}
This paper intends to show how basic counting principles from combinatorics can be translated directly into numerical solutions using spreadsheets. Relationships need not be reformulated algebraically. Additionally, the visual form of the representation will make some insights into structural relations easier to comprehend, that is, the software can be used not only to calculate numerical solutions but also to offer help for understanding mathematical concepts.
\end{abstract}

\section{Keywords}

Discrete mathematics, direct manipulation, mathematical notation, spreadsheet.

\section{INTRODUCTORY EXAMPLES FROM COMBINATORICS}

Let us start with a well-known example from combinatorics, but giving it a new twist:

If we have a group of 7 people, how many different subcommittees having exactly 3 members can we form from this group?

Let us assume the people are numbered from 1 to 7 . Then each possible committee consists of 3 different numbers in the range 1 to 7 . Of course, when writing down the members of the committee, it makes sense to arrange the numbers of the members in ascending order. So, we will talk, for example, about the committee consisting of members 1, 3, and 6. Instead of talking about subgroups or subsets of a set, we can talk about increasing sequences of a given 
length. And instead of asking for the number of possible 3 person committees we can ask for the number of increasing sequences of length 3 with the last element not being larger than 7. In such cases, it is a general mathematical technique not to solve the special case under consideration, but to study many similar problems in parallel to try to find relations between these problems, possibly yielding a solution for the general case. In our case, we want to study this problem for different upper bounds and for different lengths of the increasing sequence. The best way to summarise our results is a table (see figure 1).

We want to fill the cells of this table with the number of possible sequences. It is very easy to find the numbers for the first row of this table: If there is just one person, there is exactly 1 one-person committee, and there are no committees with more than 1 member. So we can start to fill our table (see figure 2).

Determining the number of sequences of length 1 is also very easy. The number of possible sequences of length 1 is equal to the number of numbers available, so, for example, there are 3 possible sequences of length 1 if the largest possible number is 3 . This allows us to fill the first column of our table (figure 3 ).

We have now solved our problem in all the marginal cases. How about the nonmarginal cases? Let us look at the original problem again, sequences of length 3 with a largest possible number of 7 . To get the answer for this problem we have to find the value for the grey shaded cell in our table (figure 4).

To find the value for this cell, the following observation is helpful: any sequence of length 3 with the largest possible number 6 also is a sequence of length 3 with the largest possible number 7 . We might call these sequences the 'cheating' 3 out of 7 sequences because they do not really contain the number 7 . To find the number of all 3 out of 7 sequences we have to find the number of the non-cheating or 'honest' 3 out of 7 sequences. These sequences all end with number 7. Therefore, they can be obtained by taking all 2 out of 5 sequences and adding 7 at the last place. Now let us look at our table again (figure 5).

The cell shaded in dark grey should contain the number of all 3 out of 7 sequences. The cell directly above it (shaded in light grey) should contain the number of all 3 out of 6 sequences, which also is the number of all 'cheating' 3 out of 7 sequences. The cell above and to the left should contain the number of all 2 out of 6 sequences, which is the number of all 'honest' 3 out of 7 sequences. Since the cheating and the honest sequences together yield all 3 out of 7 sequences, the number in the cell with the dark shading has to be equal to the sum of the numbers in the two cells with the light shading. We can express this relation through the diagram given in figure 6.

But this relationship is true not only for the 3 out of 7 case, but for all nonmarginal cases in our table. For each nonmarginal case, i.e. for each number of sequences with the possible largest number at least 2 and the length of the sequence at least 2 , we can divide all the sequences in 2 classes, the cheating sequences and the honest sequences. The number of cheating sequences is the number in the cell above the cell under investigation (same length of sequence, largest possible number one less). The number of the honest sequences is the 
number in the cell above and to the left (sequence shorter by one element, largest possible number one less). Therefore, the values in all the nonmarginal cells in our table can be calculated by adding the value from the cell above and the value from the cell above and to the left. The topmost and leftmost cell for which this formula has to be applied is the one for 2 out of 2 sequences (see figure 7).

As indicated in figure 8 the same formula has also to be applied to all the cells to the right and below this cell.

\begin{tabular}{|r|r|r|r|r|r|r|r|}
\hline $\begin{array}{r}\text { Largest } \\
\text { possible } \\
\text { number }\end{array}$ & \multicolumn{7}{|c|}{ Length of sequence } \\
\hline & 1 & 2 & 3 & 4 & 5 & 6 & 7 \\
\hline 1 & & & & & & & \\
\hline 2 & & & & & & & \\
\hline 3 & & & & & & & \\
\hline 4 & & & & & & & \\
\hline 5 & & & & & & & \\
\hline 6 & & & & & & & \\
\hline 7 & & & & & & & \\
\hline
\end{tabular}

\section{Figure 1}

\begin{tabular}{|r|r|r|r|r|r|r|r|}
\hline $\begin{array}{r}\text { Largest } \\
\text { possible } \\
\text { number }\end{array}$ & \multicolumn{7}{|c|}{ Length of sequence } \\
\hline & 1 & 2 & 3 & 4 & 5 & 6 & 7 \\
\hline 1 & 1 & 0 & 0 & 0 & 0 & 0 & 0 \\
\hline 2 & 2 & & & & & & \\
\hline 3 & 3 & & & & & & \\
\hline 4 & 4 & & & & & & \\
\hline 5 & 5 & & & & & & \\
\hline 6 & 6 & & & & & & \\
\hline 7 & 7 & & & & & & \\
\hline
\end{tabular}

Figure 3

\begin{tabular}{|c|c|c|c|c|c|c|c|}
\hline \multirow{2}{*}{\begin{tabular}{|l} 
Largest \\
possible \\
number
\end{tabular}} & \multicolumn{7}{|c|}{ Length of sequence } \\
\hline & 1 & 2 & 3 & 4 & 5 & 6 & \\
\hline 1 & 1 & 0 & 0 & 0 & 0 & 0 & 0 \\
\hline 2 & 2 & & & & & & \\
\hline$\sqrt{3}$ & 3 & & & & & & \\
\hline 4 & 4 & & & & & & \\
\hline 5 & 5 & & & & & & \\
\hline 6 & 6 & & & & & & \\
\hline & 7 & & & & & & \\
\hline
\end{tabular}

\section{Figure 5}

\begin{tabular}{|c|c|c|c|c|c|c|c|}
\hline \multirow{2}{*}{$\begin{array}{l}\text { Largest } \\
\text { possible } \\
\text { number }\end{array}$} & \multicolumn{7}{|c|}{ Length of sequence } \\
\hline & 1 & 2 & 3 & 4 & 5 & 6 & 7 \\
\hline 1 & $a 1$ & 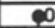 & 0 & $\overline{0}$ & 0 & 0 & $\overline{0}$ \\
\hline 2 & 2 & $8+7$ & & & & & \\
\hline 3 & $\sqrt{3}$ & & & & & & \\
\hline 4 & 4 & & & & & & \\
\hline 5 & 5 & & & & & & \\
\hline 6 & 6 & & & & & & \\
\hline 7 & 7 & & & & & & \\
\hline
\end{tabular}

Figure 7

\begin{tabular}{|c|c|c|c|c|c|c|c|}
\hline \multirow{2}{*}{\begin{tabular}{|l} 
Largest \\
possible \\
number
\end{tabular}} & \multicolumn{7}{|c|}{ Length of sequence } \\
\hline & 1 & 2 & 3 & 4 & 5 & 6 & \\
\hline 1 & 1 & 0 & 0 & 0 & 0 & 0 & \\
\hline 2 & & & & & & & \\
\hline$\sqrt{3}$ & & & & & & & \\
\hline 4 & & & & & & & \\
\hline 5 & & & & & & & \\
\hline 6 & & & & & & & \\
\hline 7 & & & & & & & \\
\hline
\end{tabular}

Figure 2

\begin{tabular}{|r|r|r|r|r|r|r|r|}
\hline $\begin{array}{r}\text { Largest } \\
\text { possible } \\
\text { number }\end{array}$ & \multicolumn{7}{|c|}{ Length of sequence } \\
\hline & 1 & 2 & 3 & 4 & 5 & 6 & 7 \\
\hline 1 & 1 & 0 & 0 & 0 & 0 & 0 & 0 \\
\hline 2 & 2 & & & & & & \\
\hline 3 & 3 & & & & & & \\
\hline 4 & 4 & & & & & & \\
\hline 5 & 5 & & & & & & \\
\hline 6 & 6 & & & & & & \\
\hline 7 & 7 & & & & & & \\
\hline
\end{tabular}

Figure 4

\begin{tabular}{|r|r|r|r|r|r|r|r|}
\hline $\begin{array}{r}\text { Largest } \\
\text { possible } \\
\text { number }\end{array}$ & \multicolumn{7}{|c|}{ Length of sequence } \\
\hline & 1 & 2 & 3 & 4 & 5 & 6 & 7 \\
\hline 1 & 1 & 0 & 0 & 0 & 0 & 0 & 0 \\
\hline 2 & 2 & & & & & & \\
\hline 3 & 3 & & & & & & \\
\hline 4 & 4 & & & & & & \\
\hline 5 & 5 & & & & & & \\
\hline 6 & 6 & 0 & & & & & \\
\hline 7 & 7 & & 4. & & & & \\
\hline
\end{tabular}

Figure 6

\begin{tabular}{|c|c|c|c|c|c|c|c|}
\hline \multirow{2}{*}{$\begin{array}{l}\text { Largest } \\
\text { possible } \\
\text { number }\end{array}$} & \multicolumn{7}{|c|}{ Length of sequence } \\
\hline & 1 & 2 & 3 & 4 & 5 & 6 & 7 \\
\hline$\overline{1}$ & a 1 & @ & 0 & 0 & 0 & 0 & 0 \\
\hline 2 & 2 & & & & & & \\
\hline 3 & 3 & & & & & & \\
\hline 4 & 4 & & & & & & \\
\hline 5 & 5 & & & & & & 愳 \\
\hline 6 & 6 & & & 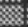 & $m$ & 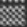 & 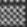 \\
\hline 7 & 7 & & $x$ & 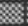 & $m$ & 18 & \\
\hline
\end{tabular}

Figure 8 
So far, we have only been talking about mathematical structure. Computer programs have not been mentioned. It turns out that the way we were analysing our combinatorics problem fits very nicely with spreadsheet programs. In a modern spreadsheet program, a formula may be entered by selecting a cell, then pressing the $=$ key, and then just clicking on the 'input' cells and entering the arithmetic operators from the keyboard. Therefore, it is very easy to create the formula for the 2 out if 2 case by selection the cell shaded in dark grey, then pressing $=$, then clicking on the cell above and to the left, then pressing + , and then clicking on the cell directly above. Of course, this point and click method creates the formula in just one cell. We need the same formula in all the cells to the right and below. At this point, one of the most important techniques of spreadsheet modelling really helps finishing our task. When a typical formula in a spreadsheet cell is copied to other cells, the relative position of the input cells to the formula cell will be preserved. In our case, copying the formula from the cell with the dark shading to the cells with the light shading will create the formula

"add the cell above and the cell above and to the left"

in all the target cells. If not explicitly declared otherwise, references to other cells in spreadsheet formulas are relative references. When a formula is copied to another cell, all these references will be moved the same way the formula is moved when it is being copied. So our mathematical model for calculating the number of subsets of a fixed size translates very easily into a spreadsheet which immediately calculates all the numbers. When we enter the formula for the 2 out of 2 cell, we immediately see the result, as in figure 9, and copying the formula 'with one sweep' immediately fills the whole table (figure 10).

\begin{tabular}{|r|r|r|r|r|r|r|r|}
\hline $\begin{array}{r}\text { Largest } \\
\text { possible }\end{array}$ & \multicolumn{7}{|c|}{ Length of sequence } \\
\cline { 2 - 8 } & 1 & 2 & 3 & 4 & 5 & 6 & 7 \\
\hline 1 & 1 & 0 & 0 & 0 & 0 & 0 & 0 \\
\hline 2 & 2 & 1 & & & & & \\
\hline 3 & 3 & & & & & & \\
\hline 4 & 4 & & & & & & \\
\hline 5 & 5 & & & & & & \\
\hline 6 & 6 & & & & & & \\
\hline 7 & 7 & & & & & & \\
\hline
\end{tabular}

Figure 9

\begin{tabular}{|r|r|r|r|r|r|r|r|}
\hline \begin{tabular}{|} 
Largest \\
possible \\
number
\end{tabular} & \multicolumn{7}{|c|}{ Length of sequence } \\
\cline { 2 - 7 } & 1 & 2 & 3 & 4 & 5 & 6 & 7 \\
\hline 1 & 1 & 0 & 0 & 0 & 0 & 0 & 0 \\
\hline 2 & 2 & 1 & 0 & 0 & 0 & 0 & 0 \\
\hline 3 & 3 & 3 & 1 & 0 & 0 & 0 & 0 \\
\hline 4 & 4 & 6 & 4 & 1 & 0 & 0 & 0 \\
\hline 5 & 5 & 10 & 10 & 5 & 1 & 0 & 0 \\
\hline 6 & 6 & 15 & 20 & 15 & 6 & 1 & 0 \\
\hline 7 & 7 & 21 & 35 & 35 & 21 & 7 & 1 \\
\hline
\end{tabular}

Figure 10

Let us reconsider what we have done so far. We started with a classical combinatorial problem, and with very basic counting principles we could establish a description of the table containing the solution for any version of the problem:

To calculate the number of different selections of fixed size out of a given set of a given size, construct a table with the number of the base set in the header column and the size of the selection in the header row. In the first column, put the row number. In the first row, put 0 (except in the first position). For all the other cells, add the numbers in the cell above and in the cell above and to the left. 
This is a very easy to understand complete description, and it works completely without using algebraic notation. This description is powerful enough to calculate the numerical solution for the usual problem sizes in very short time. The calculation method is very close to a 'natural' way of the abstract structure of the problem. This description of the solution only appeals to common sense, it is not necessary to refer to algebraic or other mathematical prerequisites.

However, translation of this solution into standard algebraic notation does provide a compact and mathematically concise description. We define a function $F(n, k)$ of 2 integer variables through the following equation:

$$
\begin{aligned}
& F(n, 1)=n \quad \text { for all } n \\
& F(1, k)=0 \quad \text { for all } k \geq 2 \\
& F(n, k)=F(n-1, k-1)+F(n-1, k) \\
& \quad \text { for } n \geq 2, k \geq 2
\end{aligned}
$$

For a mathematician it is trivial that the verbal description of the table and the algebraic formulas are equivalent, but for most people who do not have a professional mathematical background the verbal description is much easier to understand.

Looking at the algebraic definition we see that this is a recursive definition. Recursion expressed by algebraic expressions can be quite difficult to understand for many students. In the verbal description, recursion is embedded in the formulation: add the cell above and the cell to the left and above. With this phrase and the powerful visual image it is self-evident that recursion does not lead into any difficulties. The first row and the first column do not reference other cells and once these cells have their values assigned the remaining cells can be calculated row by row without any difficulties. So in this table, recursion comes in quite naturally and is coupled to a strong visual model.

So far, this alternative representation of one of the basic problems of combinatorics, combinations without repetition, helps in designing an easily understandable algorithm for the numerical solution, and this algorithm fits nicely with the spreadsheet concept of visualising relationships in a table with formulas represented by array diagrams. The structure of the problem is expressed by using relative references and the possibility of copying the formulas without additional changes.

But the power of thinking 'the spreadsheet way' does not stop here. We will use spreadsheet concepts to understand the structure of table of combinations more deeply.

Looking at our table in figure 10 we see that in the first column each cell's content can be describe as "the cell above +1 ". So adding an additional column labelled 0 (see figure 11 overleaf) containing 1 in every cell allows us to extend our formula "sum of the cells above and above and to the left" to column 1. 
82 Information and communications technologies in school mathematics

\begin{tabular}{|r|r|r|r|r|r|r|r|r|}
\hline $\begin{array}{l}\text { Largest } \\
\text { possible }\end{array}$ & \multicolumn{7}{|c|}{ Lencth of sequence } \\
\cline { 2 - 8 } number & 0 & 1 & 2 & 3 & 4 & 5 & 6 & 7 \\
\hline 1 & 1 & 1 & 0 & 0 & 0 & 0 & 0 & 0 \\
\hline 2 & 1 & 2 & 1 & 0 & 0 & 0 & 0 & 0 \\
\hline 3 & 1 & 3 & 3 & 1 & 0 & 0 & 0 & 0 \\
\hline 4 & 1 & 4 & 6 & 4 & 1 & 0 & 0 & 0 \\
\hline 5 & 1 & 5 & 10 & 10 & 5 & 1 & 0 & 0 \\
\hline 6 & 1 & 6 & 15 & 20 & 15 & 6 & 1 & 0 \\
\hline 7 & 1 & 7 & 21 & 36 & 35 & 21 & 7 & 1 \\
\hline
\end{tabular}

Figure 11

\begin{tabular}{|r|r|r|r|r|r|r|r|r|}
\hline \multirow{2}{*}{$\begin{array}{l}\text { Largest } \\
\text { possible }\end{array}$} & \multicolumn{7}{|c|}{ Length of sequence } \\
\cline { 2 - 9 } rumber & 0 & 1 & 2 & 3 & 4 & 5 & 6 & 7 \\
\hline 0 & 1 & 0 & 0 & 0 & 0 & 0 & 0 & 0 \\
\hline 1 & 1 & & & & & & & \\
\hline 2 & 1 & 2 & 1 & 0 & 0 & 0 & 0 & 0 \\
\hline 3 & 1 & 3 & 3 & 1 & 0 & 0 & 0 & 0 \\
\hline 4 & 1 & 4 & 6 & 4 & 1 & 0 & 0 & 0 \\
\hline 5 & 1 & 5 & 10 & 10 & 5 & 1 & 0 & 0 \\
\hline 6 & 1 & 6 & 15 & 2 & 15 & 6 & 1 & 0 \\
\hline 7 & 1 & 7 & 21 & 35 & 35 & 21 & 7 & 1 \\
\hline
\end{tabular}

Figure 13

\begin{tabular}{|c|c|c|c|c|c|c|c|c|}
\hline \multirow{2}{*}{\begin{tabular}{|l|} 
Largest \\
possible \\
number \\
\end{tabular}} & \multicolumn{8}{|c|}{ Length of sequence } \\
\hline & 0 & 1 & 2 & 3 & 4 & 5 & 6 & 7 \\
\hline 1 & $\bar{a}$ & 1 & $\overline{0}$ & व & $\overline{0}$ & 0 & 0 & $\overline{0}$ \\
\hline 2 & 1 & & 1 & 0 & 0 & 0 & 0 & 0 \\
\hline 3 & 1 & 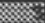 & 3 & 1 & $\overline{0}$ & 0 & 0 & 0 \\
\hline 4 & 1 & & 6 & 4 & 1 & 0 & 0 & 0 \\
\hline 5 & $\overline{1}$ & 约 & 10 & 10 & 5 & 1 & 0 & 0 \\
\hline 6 & 1 & 器 & 15 & 20 & 15 & 6 & 1 & 0 \\
\hline 7 & $\overline{1}$ & 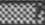 & 21 & 36 & 35 & 21 & 7 & 1 \\
\hline
\end{tabular}

Figure 12

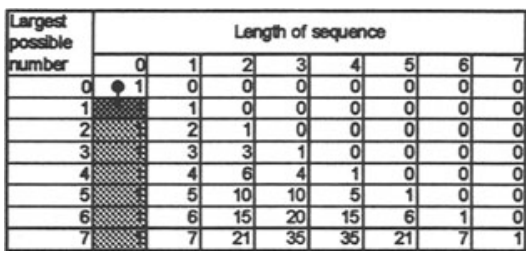

Figure 14

Again, in this expanded table, every cell except the cells in the very first row and the very first column work according to the formula "add the cell above and the cell above and to the left" (figure 12).

We can extend this table once again by adding a row labelled 0 with 1 in the first column and 0 everywhere else (figure 13). As illustrated in this image, the extended table again preserves the structure "add the cell above and the cell above and to the left" in every non-marginal cell. Compared to the original table the newer one is simpler since the first column consists only of $1 \mathrm{~s}$.

The structure of this column can easily be describe as "the just take the cell above" and this can be visualised again as indicated in figure 14.

So far, we have used the arrow notation to represent the formulas in each of the cell. We selected one sink and represented all sources flowing into the same sink. We also might say that we were looking at the mathematical structure from the consumers point of view. We can also look at the structure from the producers point of view. Expressed differently, we can say the we trace each cell with regard to where it is used as an input. Doing this we immediately see that each cell's content migrates down twice, once into the cell directly below, and once into the cell below and to the right. We show two examples in the table in figure 15. Each cell migrates down in the next row exactly twice. Therefore, row sums will double from row to row. Since the sum of row 0 is 1 , we just proved that the sum of row $n$ is $2^{n}$. The proof is very convincing and non-technical, looking at the arrows one immediately sees the validity of the assertion. We can use the "chase the arrows" method to derive further equations for the numbers in our table. 
As indicated in figure 16, the basic fact is that the dark cell is the sum of the two light cells. Marking the predecessors of the light cell to the right, i.e. marking the two cells yielding the sum for this cell. Resolving the predecessors of the right one of the two light cells in figure 16 we get we get that shown in figure 17.

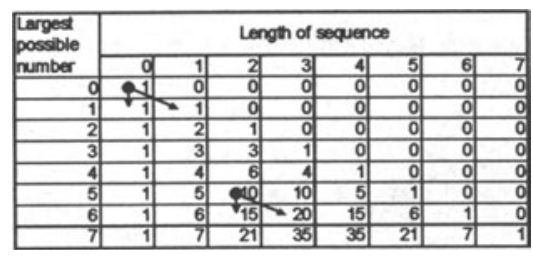

Figure 15

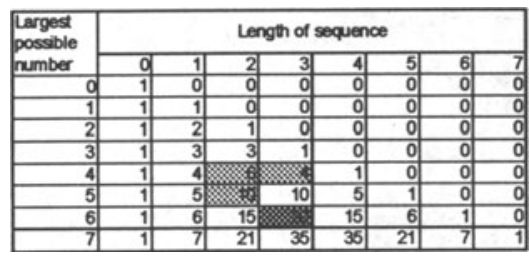

Figure 17

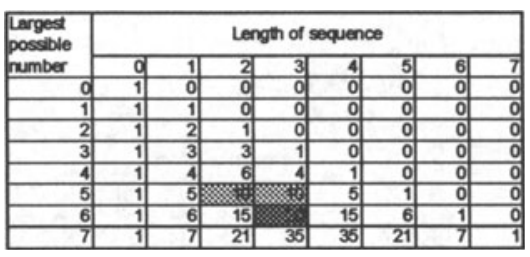

Figure 16

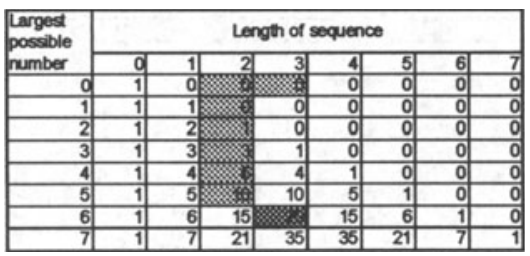

Figure 18

Continuing this predecessor chasing up until we reach the top margin produces the result shown in figure 18. Since the right topmost cell in this chain always contains 0 , we also can omit it, and therefore we see that in our table the number in each nonmarginal cell is equal to a sum of the column left to the cell under consideration. This sum extends from the very first row the row just above the cell investigated. Again, we just proved a theorem about the numbers in our table. Algebraically speaking we proved the following:

$$
F(n, k)=\sum_{i=0}^{n-1} F(i, k-1) \quad \text { for } n \geq 1, k \geq 1
$$

Using the same method of arrow chasing but always replacing the left cell of a pair of predecessors we get the pattern shown in figure 19.

\begin{tabular}{|c|c|c|c|c|c|c|c|c|}
\hline \multirow{2}{*}{$\begin{array}{l}\text { Largest } \\
\text { possible } \\
\text { number }\end{array}$} & \multicolumn{8}{|c|}{ Length of sequence } \\
\hline & a & 1 & 2 & 3 & 4 & 5 & 6 & 7 \\
\hline 0 & 1 & 0 & 0 & 0 & 0 & 0 & 0 & 0 \\
\hline 1 & 1 & 1 & 0 & 0 & 0 & 0 & 0 & 0 \\
\hline 2 & 3 & 2 & 1 & 0 & 0 & 0 & 0 & 0 \\
\hline 3 & 1 & $\overline{3}$ & 3 & 1 & 0 & 0 & 0 & 0 \\
\hline 4 & 1 & 4 & 8 & 4 & 1 & 0 & 0 & 0 \\
\hline 5 & 1 & 5 & 10 & ${ }^{\prime 2}$ & 5 & 1 & 0 & 0 \\
\hline 6 & 1 & 6 & 15 & $8 x$ & 15 & 6 & 1 & 0 \\
\hline 7 & 1 & $\overline{7}$ & 21 & 35 & 35 & 21 & 7 & \\
\hline
\end{tabular}

Figure 19 
84 Information and communications technologies in school mathematics

So each non-marginal cell is the sum of all the cells forming a diagonal starting directly above the cell under consideration and extending to the left margin of the table. Algebraically expressed this is

$$
F(n, k)=\sum_{i=0}^{k} F(n-k+i-1, i) \text { for } n \geq 1, n \geq k \geq 1
$$

Sometimes this theorem is called "hockey stick theorem", the picture given in figure 19 makes it clear why.

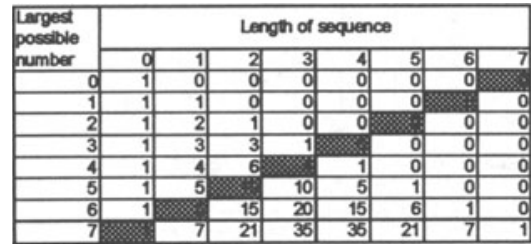

Figure 20

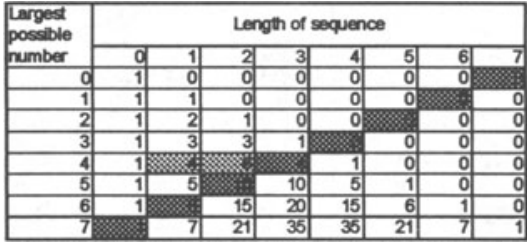

Figure 21

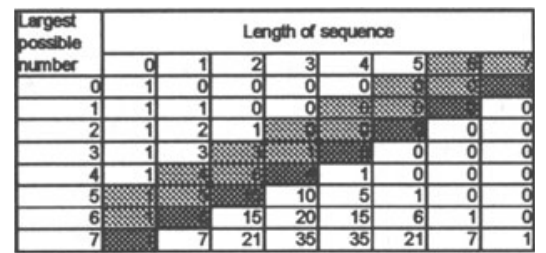

Figure 22

Let us look at one more structural property of our table. We start with a left bottom right top diagonal (see figure 20). Selecting one of the shaded cells and marking its predecessors yields the example given in figure 21, and marking the predecessors of all the cells shaded dark yields the result in figure 22. The sum of all the cells shaded dark is equal to the sum of all their predecessors, therefore the sum of the diagonal shaded dark is equal to the sum of the two diagonals shaded light.

So, if we define

$$
S(n)=\sum_{i=0}^{n} F(n-i, i)
$$

we just proved that

$$
S(n)=S(n-1)+S(n-2) \text { for } n \geq 2
$$

Since additionally we have

$$
S(0)=S(1)=1
$$

we just proved that the sum of the diagonals in our table yield the Fibonacci numbers. 
It is important to notice that for all these considerations we did not use algebraic expressions explicitly when we were analysing the mathematical structure, on the other hand, algebraic 'thinking' was implicit in the derivations. We only used algebraic notation to express the results we already knew and understood the way it is usually done in mathematics. In the next section we will study the implications of this approach.

\section{VISUALISING STRUCTURE OR ALGEBRAIC REPRESENTATION}

The key idea in the presentation of the examples in the previous section was representing mathematical relations between function values by spatial connections. This idea by itself is neither new nor tightly bound to computers. Pascal's triangle is well known, and this kind of representation has been mentioned in mathematics classes again and again. But it seems that the full power of this approach has not been realised.

Of course, all the examples in the previous section dealt with the binomial coefficients. The twist was that we did not use the most common way of expressing these numbers, the quotient of factorials,

at all.

$$
F(n, k)=\left(\begin{array}{l}
n \\
k
\end{array}\right)=\frac{n !}{k !(n-k) !}
$$

With this formula, given $n$ and $k$, one can immediately calculate the value of the function, i.e. the answer to the question. While this calculation is performed, manually or with just a pocket calculator, or even with a computer and one of the common procedural programming languages, there is no need to refer to any other value of this function. This method of performing the calculation, however, does not represent very well the counting principles we used when we set up the table with the structural relationships. So in the spread sheet context, understanding the basic principles is quite separate from performing the actual calculations. With a spreadsheet program, things change because it is possible to set up the whole table in the same time needed to perform the calculation with a calculator or a procedural computer program. The visual structure we used for expressing the mathematical structure is there from the very beginning, and is used in a natural way by creating a formula with relative references just once and the copying it to a whole range of cells. So through using a spreadsheet a context which originally was useful for understanding but not very practical for performing calculations suddenly becomes 'computationally feasible' also. It is a well established fact that the computer can help with mathematics learning because it is possible to calculate realistic examples with varying inputs studying the effects on the results of the calculation. In our case, the computer plays a somewhat different role: it turns a conceptual model into a practical calculation device. Additionally, studying the relations between the numbers in the table 
provides the opportunity for deriving more insights into the structure. Here again the computer can play an important role for understanding.

The latest version of spreadsheet programs-Microsoft Excel for exampleoffer tools to select a cell and highlight its predecessors automatically. In Excel 97, double clicking on a cell will show the cell formula with all the references to other cell colour coded, and the cells corresponding to these references will be coloured accordingly, so one gets an immediate visual representation of the calculation a cell is performing. Earlier versions of Excel offered the Auditor toolbar. Selecting a cell and applying the tools from this toolbar draws arrows similar to the ones we were using in the graphical representations in the previous section. The original intent for creating these tools was creating debugging tools for designers of large spreadsheets. But it turns out that these tools are extremely powerful in a didactical context, they can help building visual structures very quickly, and they are extremely easy to use once one know how to access them.

Using this kind of tool also shows how computer-based tools in general and spreadsheets in particular can help to create new representations of mathematical objects and relations. For hundreds of years, the working medium of mathematics has been the printed or written document. So we have become used to the fact that mathematics always is presented in a static medium. In a few decades, creating mathematical movies has been possible and some very impressive tools to help with understanding mathematics have been created using this dynamic medium. For everyday life, and for the ordinary person working with mathematics, it still has been almost impossible to create this kind of tool. Now with computers being accessible in many situations connected with mathematical learning, it is possible to use dynamic representation and animated visual aids in the everyday context of mathematical learning.

\section{CALCULATORS, COMPUTERS, SOFTWARE, AND MATHEMATICAL LEARNING (A HIGHLY PERSONAL VIEW)}

Each time a new tool for mathematics education appears, everything seems to be changing. When pocket calculators appeared, mental arithmetic (which in many cases is really paper and pencil arithmetic) became less important. With programming languages, many more problems could be tackled at the same time, and output could be nicely structured. So experimenting as a tool to 'find out' was brought into mathematics. In addition, the concept of algorithm became an object of study in mathematics.

Then came spreadsheets which have been adopted in mathematics education in many different areas, for example, see Arganbright (1985, 1995), Neuwirth (1990, 1992) and Smith (1995). Spreadsheet programs essentially have the same computational power as traditional programming languages, but they are less heavily centred on the design and implementation of algorithms. When it is possible to describe relationships as mathematical entities, it is not necessary to 
think of the order of the calculations to be performed from the very beginning. If a system of equations is consistent, the spreadsheet program will take care of performing the necessary calculations in feasible order. Also, as soon as a formula is changed, the whole system will update itself accordingly, so practical consistency is taken care of automatically.

A further advantage of spreadsheets is that they are closer to 'natural language' than procedural programming languages. Let us illustrate this. In everyday life one would say: to get the result you need, just add the number to the left and the number one more step left. In a spreadsheet, one would create the formula for this result by just clicking with the mouse. To avoid a fundamental misunderstanding here: a finished spreadsheet with the formulas in common spreadsheet notation $\left(=\mathrm{A} 1{ }^{\star} \mathrm{C} 2\right)$ is not closer to natural language nor to an everyday approach to explaining mathematical relations. The point and click method of creating formulas in a spreadsheet is what makes spreadsheets so easy to use if one has a basic understanding of the structure of the problem to be analysed. Mildly exaggerating one might say that spreadsheets automatically translate a gesture way of describing mathematics into a semi-algebraic notation. One could even refer to this way of building mathematical representations as 'gesture mathematics'. Gestures are highly dynamic, and with computers it is also easy to make the process of building a mathematical model a dynamic experience. This very fact implies that static documents like this paper cannot faithfully represent the actual experience. A more detailed discussion of these aspects of spreadsheets as a representational medium can be found in Neuwirth (1995, 1996).

Some examples aiming to demonstrate how this can be accomplished, and some ideas on how to use spreadsheets in mathematics, statistics, and science education can be found on the World Wide Web at

http://sunsite.univie.ac.at/Spreadsite

This Web site also offers a large list of reading material and further resources for spreadsheets.

\section{REFERENCES}

Arganbright, D. (1985). Mathematical Applications of Electronic Spreadsheets. New York: McGraw-Hill.

Arganbright, D (1995). Practical Handbook of Spreadsheet Curves and Geometric Constructions. Boca Raton: CRC Press.

Neuwirth, E (1990). Visualizing Correlation with Spreadsheet, Teaching Statistics, 12, 86-89. Republished in The Best of Teaching Statistics, 1994, $110-113$. 
Neuwirth, E. (1992). Tabellenkalkulation und Wahrscheinlichkeitsrechnung: Experimentelle und Strukturelle Überlegungen. In R. Mittermeir, E. Kofler und H. Steinberger (eds.) Informatik in der Schule-Informatik für die Schule $S$. Universitaet Klagenfurt, Klagenfurt, 172-184.

Neuwirth, E. (1995). Visualizing structural and formal relationships with spreadsheets. In A. DiSessa, C. Hoyles and R. Noss, The Design of Computational Media to Support Exploratory Learning. New York: Springer-Verlag, 155-174.

Neuwirth, E. (1996). Spreadsheets: Helpful for Understanding Mathematical Structures The Computing Teacher, 89(3), 252-254.

Smith, R. (1995). Applications of Spreadsheets in Calculus. In Innovative Use of Technology for Teaching and research in Mathematics: Proceedings of the First Asian Technology Conference in Mathematics. The Association of Mathematics Educators, Singapore, 491-500.

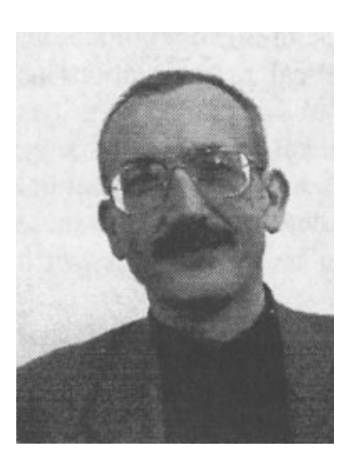

Erich Neuwirth teaches mathematics, statistics, and computer science at the University of Vienna. He has been visiting professor in the United States (Northeastern University, University of California at Davis) and Japan (National Institute of Multimedia Education). He is a member of international expert groups on computers in mathematics education, and he has acted as an advisor to the Austrian Ministry of Education. He has published many articles on using software in mathematics education, and has recently finished a multimedia project on the mathematical foundations of musical tuning systems, which won the 1996 European Academic Software Award. 